\title{
Identifying risk factors of recurrence for clinically node negative papillary thyroid carcinoma with pathologic N1a
}

\author{
Young Jae Ryu, Jin Seong Cho* ${ }^{*}$, Min Ho Park and Jung Han Yoon
}

\begin{abstract}
Background: Whether or not to perform prophylactic central lymph node dissection (CLND) in the case of clinically node-negative papillary thyroid cancer (PTC) is controversial. The purpose of this study was to investigate the risk factors for recurrence in clinically node-negative PTC patients who underwent total thyroidectomy plus bilateral central neck dissection and was verified pathologic N1a.

Methods: We retrospectively reviewed the medical records of 1082 PTC patients who underwent total thyroidectomy and prophylactic bilateral CLND between January 2004 and December 2012. We used Cox-proportional hazard regression analyses in order to explore potential predictive factors for recurrence.

Results: During a median follow-up (range) of 78 (12-158) months, recurrence occurred in 62 (5.7\%) patients. Main tumor size more than $1 \mathrm{~cm}$, gross extrathyroidal extension (ETE), positive lymph node (LN) more than 3, and LN ratio $>0.5$ were all significantly associated with recurrence according to univariate analysis. In model I multivariate analysis (tumor size, gross ETE, LN ratio), LN ratio > 5 (hazards ratio [HR], 4.794; 95\% confidence interval [Cl], 2.674-8.595; $p<0.001$ ) was found to be predictive of recurrence. Gross ETE (HR, 1.794; 95\% Cl, 1.024-3.143; $p=0.041)$ and positive LN more than 3 $(\mathrm{HR}, 2.505 ; 95 \% \mathrm{Cl}, 1.513-4.146 ; p<0.001)$ were predictors for recurrence in model II multivariate analysis (tumor size, gross $E T E$, the number of positive $L N$ ).

Conclusions: We recommend that surgeons try to focus completely on performing prophylactic CLND for patients with suspicious gross ETE during preoperative evaluation. Close monitoring and thorough management are needed for clinically node-negative PTC patients with $L N$ ratio of more than 0.5 and more than 3 positive $L N$ in the central compartment.
\end{abstract}

Keywords: Papillary thyroid cancer, Prophylactic central neck dissection, Recurrence

\section{Background}

Papillary thyroid cancer (PTC) is the most common histologic type of thyroid cancer. Although the involvement of lymph node (LN) in PTC is high upon the initial diagnosis due to the lymphogenous spread pattern, PTC patients have better survival outcomes than patients with other thyroid malignancies, with an over $95 \%$ survival rate in 10 years and $93 \%$ survival in 20 years [1]. On the other hand, the recurrence rate during follow-up can be up to $30 \%$ in PTC $[2,3]$. One study reported that up to $90 \%$ of PTC patients with recurrence had LN

\footnotetext{
* Correspondence: sabiston@hotmail.com

Department of Surgery, Chonnam National University Medical School, 42 Jebong-ro, Dong-gu, Gwangju 61469, Republic of Korea
}

metastasis at first operation, and that the presence of $\mathrm{LN}$ metastases is associated with mortality due to thyroid cancer [4]. The detection of LN metastasis prior to surgery is somewhat difficult. The diagnosis of micro PTC that is below $1 \mathrm{~cm}$ in tumor size has become easier through the introduction of high resolution ultrasonography (US). From the perspective of detecting LN, distinguishing suspicious LN in the central compartment is harder than in the lateral compartment. Besides, the sensitivity of US and computed tomography (CT) is lower for detecting $\mathrm{LN}$ in the central compartment because of the anatomical location (proximity to the thyroid and trachea) [5].

(C) The Author(s). 2019 Open Access This article is distributed under the terms of the Creative Commons Attribution 4.0 International License (http://creativecommons.org/licenses/by/4.0/), which permits unrestricted use, distribution, and reproduction in any medium, provided you give appropriate credit to the original author(s) and the source, provide a link to the Creative Commons license, and indicate if changes were made. The Creative Commons Public Domain Dedication waiver (http://creativecommons.org/publicdomain/zero/1.0/) applies to the data made available in this article, unless otherwise stated. 
Therapeutic central LN dissection (CLND) using the same incision is acceptable for the management of clinically node-positive PTC; however, performing prophylactic CLND for clinically node-negative PTC remains controversial. According to recent guidelines from the American Thyroid Association (ATA), prophylactic CLND should be considered in patients with clinically central node-negative PTC who have advanced primary tumors (T3 or T4) or who are clinically node-positive in the lateral compartment, but it is not recommended for patients with small (T1 or T2), noninvasive, or clinically node-negative PTC [6]. In addition to the ATA guidelines, the European Society of Endocrine Surgeons has suggested that prophylactic CLND is appropriate for patients aged 45 years and older or 15 years and younger, male gender, and patients with bilateral or multifocal tumors [7]. There is still a lack of evidence showing that the removal of as much LN as possible gives rise to better survival outcomes. The ultimate goal of prophylactic CLND is not the improvement of survival, but the reduction of reoperation that might cause higher rates of complications as well as the facilitation of postoperative follow-up using thyroglobulin (Tg). In addition, prophylactic CLND is helpful for achieving accurate nodal stage and decision-making of radioactive iodine (RAI) therapy. Even when LN metastases are revealed, clinicians can consider the omission of RAI therapy for patients with lower risk of recurrence in PTC.

It is generally accepted that the extending pattern of LNs in PTC begins from the central compartment and progresses toward the lateral compartment. The patients who underwent prophylactic CLND had subclinical LN metastasis up to $50 \%[8,9]$. Several studies have focused on the efficacy of prophylactic CLND; however, there have been few studies on PTC patients who underwent prophylactic CLND with confirmed pathologic N1a. Hence, the aim of this study was to investigate the risk factors for recurrence in clinically node-negative PTC patients who underwent total thyroidectomy plus bilateral central neck dissection with verified pathologic N1a.

\section{Methods}

\section{Patients}

We reviewed the medical records of 9135 patients who underwent thyroid surgery at Chonnam National University Hwasun Hospital between January 2004 and December 2012. Exclusion criteria were as followings: patients who were under 15 years old, who underwent completion thyroidectomy due to recurrence of any histologic type, who underwent thyroidectomy due to benign thyroid disease or other thyroid malignancy besides PTC, who underwent lateral neck dissection at first surgery, who were clinically node-positive in the central and lateral compartment, who did not undergo CLND, who were confirmed as having pathologic N0, who did not achieve R0 resection, who had distant metastasis upon first diagnosis, who had abnormal thyroid function test results prior to operation, who had less than a one year follow-up period, who experienced recurrence within one year after surgery, and who had other malignancies pre- or postoperatively. We enrolled a total of 1082 patients who underwent total thyroidectomy and prophylactic bilateral CLND in this study. All patients were carefully reviewed preoperative neck US and neck CT for the evaluation of the LNs in the central neck compartment. This retrospective study was approved by our hospital's institutional review board and permission is required to access the data.

All patients were confirmed as having Bethesda category V (154 patients, 14.2\%) or VI (928 patients, 85.8\%) PTC through preoperative fine needle aspiration cytology (FNAC). We exhaustively collected patients' clinicopathological information, LN ratio (number of metastatic LNs divided by number of harvested LNs), complications related to operation, and recurrence. Cancer staging followed the recent recommendations outlined by American Joint Committee on Cancer (AJCC) [10].

\section{Operation and postoperative follow-up}

All patients underwent total thyroidectomy and comprehensive bilateral CLND in level VI (Delphian, pre- and paratracheal, and/or paraesophageal node) and level VII (upper mediastinal node). All surgeries were performed by the same thyroid surgical team. Specimens were sent to the pathologic department and meticulously examined by experienced pathologists. Based on the tumor and node characteristics, patients received $30-150 \mathrm{mCi}$ of RAI therapy at two to three months following surgery. However, the omission of RAI therapy was considered in some cases because of the clinician's decision or patient's refusal. The interval of follow-up was three to six months for the first three years and thereafter annual. All patients received regular physical examinations, neck US, chest radiography, measurement of serum-free thyroxine and thyrotropin, $\mathrm{Tg}$, and anti-Tg antibody concentrations every visit. If necessary, neck CT, wholebody iodine scanning, and 18F-fluorodeoxyglucose positron emission tomography $\mathrm{CT}$ were performed for further evaluation. We defined recurrence as structural recurrence, which was evaluated using image modalities and histologic examinations. Suspicious structural recurrence was confirmed with FNAC, if accessible. Reoperation followed by RAI therapy was applicable to most patients with structural recurrence; however, if the lesion of recurrence was unresectable distant organ, RAI therapy was considered as the best treatment option rather than reoperation. 


\section{Complications}

We determined wound infections to be cases requiring local treatment including antibiotics because of cellulitis or those needing incision and drainage because of deep neck infection. The time of distinction between transience and permanence, which is applicable to hypoparathyroidism and recurrent laryngeal nerve injury, was six months after the initial surgery. According to our institution's protocols, the measurement of parathyroid hormone (PTH) with the level of total calcium and ionized calcium was examined in six hours, one day, and two days postoperatively for patients who underwent thyroid surgery. We defined permanent hypoparathyroidism as below-normal serum PTH with low level of calcium that should be maintained calcium and vitamin D supplementation more than 6 months after surgery. Unfortunately, the patients enrolled in this study did not routinely undergo preoperative flexible laryngoscopy. However, selective flexible laryngoscopy was performed for patients who had suspicious gross ETE towards tracheoesophageal groove or symptoms related to voice changes preoperatively, who developed voice changes or had suspicious of recurrent laryngeal nerve injury postoperatively. We defined postoperative bleeding to be cases requiring conservative treatment or reoperation.

\section{Statistical analysis}

The primary end point was any structural recurrence in the loco-region or distant organs. Recurrence-free survival (RFS) was defined as the period between the first operation and the detection of recurrence. Continuous variables are presented as median (range) or mean (standard deviation); categorical variables are presented as number (percent). We used a univariate Coxproportional hazards model in order to analyze the relationships between the clinicopathological variables and RFS. We also conducted multivariate Cox-proportional hazards regression analyses by means of backward elimination using the variables with $p<0.05$ in the univariate analyses. The receiver operating characteristic curve was used to calculate an optimal LN ratio cutoff. The Kaplan-Meier curve with log-rank test was utilized for comparison of RFS according to LN ratio, number of metastatic LN, and ETE. All statistical analyses were performed using SPSS version 23.0 (IBM Inc., Armonk, NY, USA) and defined statistical significance to be $\mathrm{p}$ less than 0.05 .

\section{Results}

\section{Patients' demographics}

Of the total 1082 patients, 213 (19.7\%) were male. The median (range) age was 46 years (15-75), and 260 patients $(24.0 \%)$ were over 55 . The mean primary tumor size was $1.15 \mathrm{~cm}$ (standard deviation, $0.74 \mathrm{~cm}$ ), and 430 patients (39.7\%) had a main tumor more than $1 \mathrm{~cm}$ in size, while $652(60.3 \%)$ patients had microcarcinoma. There were 906 patients $(83.7 \%)$ with tumors limited to the thyroid (T1, T2, T3a), 119 (11.0\%) with tumors with gross ETE invading only strap muscles (T3b), and 57 (5.3\%) with stage T4a tumors. Six hundred thirty-five (58.7\%) patients had more than five harvested central lymph nodes. Three hundred thirty-one (30.6\%) patients and $242(22.4 \%)$ patients had tumor multifocality and bilaterality, respectively. We observed chronic lymphocytic thyroiditis in 334 (30.9\%) patients and lymphovascular invasion in $23(2.1 \%)$ patients. Nine hundred eighty-six (91.1\%) patients were administered postoperative RAI therapy. According to AJCC TNM stage, 950 (87.7\%) patients belonged to stage I; 116 (10.7\%), stage II; and $16(1.5 \%)$, stage III. The median follow-up (range) was 78 (12-158) months (Table 1).

During follow-up, recurrence occurred in 62 (5.7\%) patients. The mean time to recurrence was 36 months (range, 12-102 months). Of these 62 patients, 19 only had recurrence in the operative bed (15 patients) or central neck compartment (four patients), while 32 only had it in the lateral neck compartment. Nine patients had recurrence in the central and lateral compartments simultaneously. Two patients had distant metastasis confined to the lung.

\section{Univariate analyses according to recurrence}

Univariate analysis using the Cox-proportional hazards regression model for all patients showed that main tumor size more than $1 \mathrm{~cm}$ (vs. $\leq 1 \mathrm{~cm} ; p=0.047$ ), gross ETE (vs. no gross ETE; $p=0.018$ ), positive LN more than 3 (vs. $\leq 2 ; p<0.001$ ), and $\mathrm{LN}$ ratio $>0.5$ (vs. $\leq 0.5$; $p<0.001$ ) were all significantly associated with recurrence. There were no significant effects of age, gender, multifocality, bilaterality, LVI, CLT, number of harvested LNs, or TNM stage (Table 2, Fig. 1).

\section{Multivariate analyses according to recurrence}

We divided the number of positive $\mathrm{LN}$ and $\mathrm{LN}$ ratio to avoid the interference in nodal factors. Multivariate Cox-proportional hazards regression analyses revealed that LN ratio $>5$ (hazards ratio [HR], 4.794; 95\% confidence interval $[\mathrm{CI}], 2.674-8.595 ; p<0.001)$ was a predictor of recurrence in model I (tumor size, gross ETE, $\mathrm{LN}$ ratio), and that gross ETE (HR, 1.794; 95\% CI, $1.024-3.143 ; p=0.041$ ) and positive LN more than 3 (HR, 2.505; 95\% CI, 1.513-4.146; $p<0.001$ ) were predictors of recurrence in model II (tumor size, gross ETE, the number of positive LN) (Table 3).

\section{Complications}

Of the total 1082 PTC patients, no patient suffered from wound infection. The incidence rates of transient and 
Table 1 Patients' demographics

\begin{tabular}{|c|c|}
\hline Variables & Number (percent) \\
\hline $\mathrm{Age}^{\S}$ & 46.0 years $(15-75)$ \\
\hline$<55$ years & $822(76.0)$ \\
\hline$\geq 55$ years & $260(24.0)$ \\
\hline \multicolumn{2}{|l|}{ Sex } \\
\hline Female & $869(80.3)$ \\
\hline Male & $213(19.7)$ \\
\hline \multicolumn{2}{|l|}{ Underlying disease } \\
\hline Hypertension & $183(16.9)$ \\
\hline Diabetes & $71(6.6)$ \\
\hline Tumor size & $1.15 \mathrm{~cm} \pm 0.74$ \\
\hline$\leq 1 \mathrm{~cm}$ & $652(60.3)$ \\
\hline$>1 \mathrm{~cm}$ & $430(39.7)$ \\
\hline \multicolumn{2}{|l|}{ T stage } \\
\hline T1a & $622(57.5)$ \\
\hline $\mathrm{T} 1 \mathrm{~b}$ & $230(21.3)$ \\
\hline $\mathrm{T} 2$ & $51(4.7)$ \\
\hline Т3а & $3(0.3)$ \\
\hline $\mathrm{T} 3 \mathrm{~b}$ & $119(11.0)$ \\
\hline T4a & $57(5.3)$ \\
\hline \multicolumn{2}{|l|}{ Number of harvested lymph node } \\
\hline$\leq 4$ & $447(41.3)$ \\
\hline$\geq 5$ & $635(58.7)$ \\
\hline Multifocality & $331(30.6)$ \\
\hline Bilaterality & $242(22.4)$ \\
\hline Chronic lymphocytic thyroiditis & $334(30.9)$ \\
\hline Lymphovascular invasion & $23(2.1)$ \\
\hline \multicolumn{2}{|l|}{ TNM stage } \\
\hline Stage I & $950(87.8)$ \\
\hline Stage II & $116(10.7)$ \\
\hline Stage III & $16(1.5)$ \\
\hline Radioactive iodine therapy & $986(91.1)$ \\
\hline Recurrence & $62(5.7)$ \\
\hline follow-up $p^{\S}$ & 78 months (12-158) \\
\hline Total patients & $1082(100)$ \\
\hline
\end{tabular}

$\S$ represents median and range, ${ }^{*}$ represents mean and standard deviation

permanent hypoparathyroidism were $5.8 \%$ (63 patients) and $3.4 \%$ (37 patients), respectively; we observed transient and permanent recurrent laryngeal nerve injury in $32(3.0 \%)$ and $10(0.9 \%)$ patients. Two patients underwent reoperation due to postoperative bleeding (Table 4)

\section{Discussion}

Through this study, we aimed to determine the predictors of recurrence in 1082 clinically node-negative PTC
Table 2 Univariate Cox Regression of recurrence

\begin{tabular}{llll}
\hline Variables & HR & $95 \% \mathrm{Cl}$ & $p$ \\
\hline Age & & & \\
$\quad<55$ years & 1 & & \\
$\quad \geq 55$ years & 0.674 & $0.351-1.294$ & 0.236 \\
Sex & & & \\
$\quad$ Female & 1 & & 0.450 \\
Male & 1.258 & $0.694-2.282$ &
\end{tabular}

Tumor size

$$
\begin{aligned}
& \leq 1 \mathrm{~cm} \\
& >1 \mathrm{~cm}
\end{aligned}
$$

1.656

$1.006-2.725$

0.047

Multifocality

$$
\text { No }
$$

Yes

1.543

$0.929-2.562$

0.094

Bilaterality

No

Yes

LVI

No

Yes

0.891

$0.515-1.543$

0.891

CLT

\section{No}

Yes

0.891

$0.515-1.543$

T stage

T1/2/3a
T3b
T4a

1

1.013

$0.432-2.376$

0.976

ETE

No gross ETE
Gross ETE
Harvested LN
$\leq 4$
$\geq 5$

4.019

2.079-7.773

Positive LN

$$
\leq 2
$$

$\geq 3$

$\mathrm{LN}$ ratio

$$
\leq 0.5
$$$$
>0.5
$$

TNM stage

$$
\text { Stage I }
$$

Stage II/III

0.507

$0.184-1.398$

0.189

$\mathrm{Cl}=$ Confidence interval, $\mathrm{CLT}=$ Chronic lymphocytic thyroiditis, $\mathrm{ETE}=$ Extrathyroidal extension, $\mathrm{HR}=$ Hazards ratio, $\mathrm{LN}=\mathrm{Lymph}$ node, $\mathrm{LVI}=$ Lymphovascular invasion 

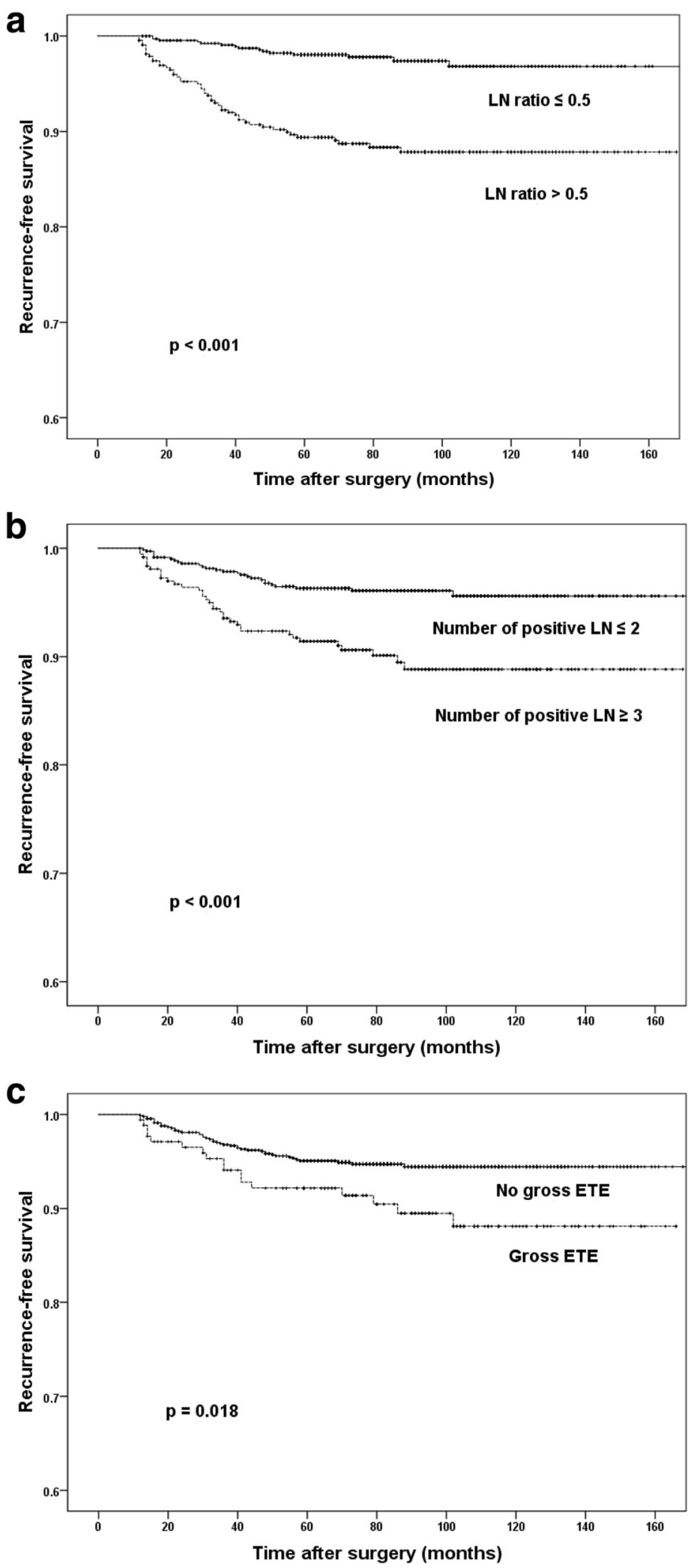

Fig. 1 Kaplan-Meier curves according to variables. LN ratio (a), Number of positive LN (b), Gross ETE (c). ETE = Extrathyroidal extension, LN = Lymph node 
Table 3 Multivariate Cox Regression of recurrence

\begin{tabular}{|c|c|c|c|c|c|c|}
\hline \multirow[t]{2}{*}{ Variables } & \multicolumn{3}{|c|}{ Model I } & \multicolumn{3}{|c|}{ Model II } \\
\hline & $\mathrm{HR}$ & $95 \% \mathrm{Cl}$ & $p$ & $\mathrm{HR}$ & $95 \% \mathrm{Cl}$ & $p$ \\
\hline \multicolumn{7}{|l|}{ Tumor size } \\
\hline$\leq 1 \mathrm{~cm}$ & 1 & & & 1 & & \\
\hline$>1 \mathrm{~cm}$ & 1.260 & $0.754-2.105$ & 0.377 & 1.354 & $0.808-2.268$ & 0.249 \\
\hline \multicolumn{7}{|l|}{ ETE } \\
\hline No gross ETE & 1 & & & 1 & & \\
\hline Gross ETE & 1.645 & $0.939-2.883$ & 0.082 & 1.794 & $1.024-3.143$ & 0.041 \\
\hline \multicolumn{7}{|l|}{ LN ratio } \\
\hline$\leq 0.5$ & 1 & & & & & \\
\hline$>0.5$ & 4.794 & $2.674-8.595$ & $<0.001$ & & & \\
\hline \multicolumn{7}{|l|}{ Positive LN } \\
\hline$\leq 2$ & & & & 1 & & \\
\hline$\geq 3$ & & & & 2.505 & $1.513-4.146$ & $<0.001$ \\
\hline
\end{tabular}

patients who underwent total thyroidectomy plus bilateral CLND and who had proven pathologic N1a. During a follow-up period of 78 months (range, 12-158), 62 patients experienced recurrence which was predicted by gross ETE and nodal factors (LN ratio $>0.5$, the number of positive $\mathrm{LN} \geq 3$ ).

There are three main strategies regarding the management of thyroid cancer: surgery, thyroid stimulating hormone suppression, and RAI ablation therapy. Of these three modalities, clinicians think that surgical completeness is the most important. Complete resection of the LN is important for surgical completeness, along with removal of the primary tumor. It is understandable that patients who are clinically node-negative do not need prophylactic lateral neck dissection; however, prophylactic CLND is still a debatable issue in terms of management in clinically node-negative PTC patients.

Prophylactic CLND may have the possibility of upstaging in considerable number of patients with PTC and induce proper $\mathrm{N}$ stage for RAI therapy. Hughes et al. found that prophylactic CLND has resulted in upstaging for one-third patients older than 45 years and

Table 4 Postoperative complications

\begin{tabular}{ll}
\hline Complication & Number (\%) \\
\hline Wound infection & 0 \\
Hypocalcemia & $63(5.8)$ \\
Transient & $37(3.4)$ \\
Permanent & \\
Recurrent laryngeal nerve injury & $32(3.0)$ \\
Transient & $10(0.9)$ \\
Permanent & $2(0.2)$ \\
\hline Bleeding &
\end{tabular}

has also resulted in the modification of dose in RAI therapy [11]. Another study reported that 30\% PTC patients with T1 tumor who underwent prophylactic CLND can be indicated for RAI therapy [12]. However, the patients with pathologic N0 following prophylactic CLND may have the advantage of low-dose RAI or no RAI therapy based on tumor features. Therefore, prophylactic CLND provides important information regarding more tailored management of RAI therapy and postoperative followup using $\mathrm{Tg}$ in PTC patients with subclinical LN metastases.

Meta-analysis reported that prophylactic CLND decrease locoregional recurrence and had the opposite relationship in terms of postoperative hypocalcemia [13, 14]. Another meta-analysis showed that the recurrence rate is $2.02 \%$ in patients who underwent thyroidectomy plus prophylactic CLND and $3.92 \%$ in patients who underwent only thyroidectomy; however, prophylactic CLND was not significantly associated with local recurrence (odds ratio $=1.05$, 95\% confidence interval 0.48 2.31) [15]. Wang et al. concluded that there were no significant differences in recurrence and long term complications between patients who underwent only total thyroidectomy and those who underwent total thyroidectomy plus prophylactic CLND [16]. Considering the optimal (maximal) outcomes and quality of life in clinically node-negative PTC patients, prophylactic CLND is an imperative topic to be considered by the thyroid surgeons. The rates of surgery-related permanent complications may be lower when not performing prophylactic CLND. When recurrence occurs, repeated thyroid surgery is more challenging due to the formation of scar tissue and distorted anatomy, which may lead to the increased risk of permanent complications. Recurrence in the central compartment for patients who did not 
undergo prophylactic CLND in the first operation is more difficult to identify than that in the lateral compartment [17]. The rates of complication are closely associated with the surgical extent and could be the reason for the curtailment of the surgical extent in PTC. Patients who undergo reoperation due to recurred PTC have a high incidence of several operations in the future and consequently do not achieve a biochemical complete response during follow-up $[18,19]$. Reoperation in the central compartment by an experienced thyroid surgeon is a safe procedure and does not increase the risk of surgical complications compared to initial prophylactic CLND [20, 21]. Previous research has reported that prophylactic CLND is safe as performed by high-volume thyroid surgeon [22, 23]. Major development in technology, such as energy devices and intraoperative neuro-monitoring may lead to lower surgery-related complications, especially reoperation.

The factors that are associated with aggressive disease in PTC are age, male gender, large primary tumor size, and PTC variant histologic type such as tall cell, columnar cell, Hurthle cell, diffuse sclerosis, and insular variant. The features of the primary tumor are one of the salient factors for predicting LN involvement in the central compartment and helpful for deciding the extent of thyroidectomy [24]. ETE has been regarded as one of the important independent prognostic factors for persistent and recurrent PTC and has been slightly changed according to the most recent AJCC staging manual. The meaning of minor ETE was excluded because of equivalent survival outcomes between patients with minor ETE and those without ETE. In addition, tumor $>4 \mathrm{~cm}$ in the greatest dimension bounded to the thyroid gland is considered T3a and gross ETE with invasion only to the strap muscles is considered T3b [10]. This present study also recognized that there was no significant association according to recurrence among patients with or without minor ETE. Also, patients with gross ETE had a higher recurrence rate than those without ETE. Therefore, meticulous CLND is needed for clinically node-negative PTC patients with gross ETE during preoperative evaluation and intraoperative finding.

In the study with unilateral PTC and clinically nodenegative patients who underwent total thyroidectomy plus bilateral CLND, authors reported $42.9 \%$ unilateral subclinical metastases in the central compartment and a high possibility of subclinical metastases if the tumor was more than $1 \mathrm{~cm}$ in size [25]. Provided that the distinction of LN involvement is possible prior to operation, surgeons can decide between therapeutic or prophylactic CLND depending on LN status; however, determining whether or not there is suspicious $\mathrm{LN}$ in the central compartment prior to surgery is very difficult. In addition, the sensitivity of preoperative US and
CT is relatively low to detect suspicious $\mathrm{LN}$ in the central compartment [26]. Some authors have suggested that intraoperative frozen section examination of the ipsilateral central LN should be the primary surgical approach option taken prior performing bilateral CLND for clinically node-negative PTC [27]. Although the introduction of this approach is relatively short for the analysis of PTC patients in our institution, it may suitable for recent trends that are changing to minimize the extent of thyroid surgery. It is generally accepted that the lymphatic spread pattern of PTC begins in the central compartment at first, followed afterward by the lateral compartment. Using this concept, some surgeon have adopted sentinel LN biopsy, which is used for melanoma and breast cancer to decide the surgical extent of $\mathrm{LN}$ intra-operatively; however, there is still a point to be overcome before applying it to PTC because of its high false negative rate [28].

In the study with clinically node-negative PTC patients during a median follow-up of 164 months, the authors revealed that $59 \%$ of total patients were confirmed N1a on postoperative pathologic result [9]. They demonstrated that age (older than 55 years), tumor size (more than $2 \mathrm{~cm}$ ), significant ETE, and the number of confirmed central LN metastases (more than 5) were associated with recurrence in the central compartment [9]. A considerable number of clinically node-negative PTC patients were confirmed as having pathologic N1a. Therefore, it is important to achieve complete removal of LN in the central compartment when performing the first thyroid surgery. Although there have been several studies regarding the efficacy of prophylactic CLND in PTC, most studies have included PTC patients with pathologic N1a and N0, and some research has classified clinically node-negative PTC patients who underwent only thyroidectomy as N0 stage $[8,29]$. This present study excluded clinically node-negative PTC with pathologic NO, as we tried to find out the predictive factors of recurrence in clinically node-negative PTC with pathologic N1a. Although subclinical LN metastasis was observed in up to $50 \%$ of clinically node-negative PTC patients, nothing has yet been determined about the number of harvested central LN when performing CLND in thyroid surgery. We did not observe an optimal number of harvested central LN in this study. According to the recently published AJCC 8th edition, NOa is defined as one or more cytologically or histologically confirmed benign lymph nodes [10]. Although we performed comprehensive CLND, some patients had a small number of central LN on the pathologic results. In order to supplement this, we additionally analyzed LN ratio. This study revealed that the number of positive central LNs and the LN ratio could be important indicators supplementing the current TNM stage. 
This study has several limitations. First, this study design is retrospective and some patients had relatively short-term follow-up period. Second, nine hundred eighty-six (91.1\%) patients underwent RAI therapy in this study; however, many of those patients were classified as low risk group, RAI therapy can potentially be omitted in such cases according to recent guidelines; we did not consider the level of stimulated $\mathrm{Tg}$ or the influence of RAI therapy. Third, we did not consider biochemical incomplete response regarding the level of $\mathrm{Tg}$ and anti-Tg antibody during postoperative follow-up. In addition, certain patients showed undetectable levels of $\mathrm{Tg}$ and anti-Tg antibody, irrespective of having structural recurrence. Fourth, we did not include the statuses of involved LNs such as size and extranodal extension. We are planning to collect sufficient clinicopathological data on long-term follow-up results in PTC patients who underwent prophylactic CLND.

\section{Conclusions}

Performing prophylactic CLND in clinically nodenegative PTC patients is a useful and efficient procedure. We recommend that surgeons try to focus on complete performing prophylactic CLND for patients with suspicious gross ETE during preoperative evaluation. Close monitoring and thorough management are needed for clinically node-negative PTC patients with more than 0.5 of $\mathrm{LN}$ ratio and more than three positive LNs in the central compartment.

\section{Abbreviations}

AJCC: American Joint Committee on Cancer; ATA: American Thyroid Association; Cl: Confidence interval; CLND: Central lymph node dissection; CLT: Chronic lymphocytic thyroiditis; CT: Computed tomography; ETE: Extrathyroidal extension; FNAC: Fine needle aspiration cytology; HR: Hazards ratio; LN: Lymph node; LVI: Lymphovascular invasion; PTC: Papillary thyroid cancer; PTH: Parathyroid hormone; RAI: Radioactive iodine; RFS: Recurrence-free survival; Tg: Thyroglobulin; US: Ultrasonography

\section{Acknowledgements}

Not applicable.

\section{Authors' contributions}

YJR is the main author of the manuscript and have substantial contributions to the study design and the data analysis. MHP and JHY helped the data collection. JSC involved in the study design and supervision. All authors read and approved the final manuscript.

\section{Funding}

This study was supported by a grant (CRI 18 90*-1 BCR118026) from Chonnam National University Hospital Biomedical Research Institute.

\section{Availability of data and materials}

The datasets used and analyzed during the current study available from the corresponding author on reasonable request.

\section{Ethics approval and consent to participate}

This study was approved by the institutional review board in Chonnam National University Hwasun Hospital. Patient informed consent was waived because of the retrospective nature of the study.
Consent for publication

Not applicable.

\section{Competing interests}

The authors declare that they have no competing interests.

Received: 27 July 2018 Accepted: 24 June 2019

Published online: 05 July 2019

\section{References}

1. Wang Q, Chu B, Zhu J, Zhang S, Liu Y, Zhuang M, et al. Clinical analysis of prophylactic central neck dissection for papillary thyroid carcinoma. Clin Transl Oncol. 2014;16:44-8.

2. Hay ID, Thompson GB, Grant CS, Bergstralh EJ, Dvorak CE, Gorman CA, et al. Papillary thyroid carcinoma managed at the Mayo Clinic during six decades (1940-1999): temporal trends in initial therapy and long-term outcome in 2444 consecutively treated patients. World J Surg. 2002;26:879-85.

3. Sippel RS, Chen H. Controversies in the surgical management of newly diagnosed and recurrent/residual thyroid cancer. Thyroid. 2009;19:1373-80.

4. Lundgren Cl, Hall P, Dickman PW. Zedenius J clinically significant prognostic factors for differentiated thyroid carcinoma: a population-based, nested case-control study. Cancer. 2006;106:524-31.

5. Ahn JE, Lee JH, Yi JS, Shong YK, Hong SJ, Lee DH, et al. Diagnostic accuracy of $\mathrm{CT}$ and ultrasonography for evaluating metastatic cervical lymph nodes in patients with thyroid cancer. World J Surg. 2008:32:1552-8.

6. Haugen BR, Alexander EK, Bible KC, Doherty GM, Mandel SJ, Nikiforov YE, et al. 2015 American Thyroid Association management guidelines for adult patients with thyroid nodules and differentiated thyroid Cancer: the American Thyroid Association guidelines task force on thyroid nodules and differentiated thyroid Cancer. Thyroid. 2016;26:1-133.

7. Sancho JJ, Lennard TW, Paunovic I, Triponez F, Sitges-Serra A. Prophylactic central neck disection in papillary thyroid cancer: a consensus report of the European Society of Endocrine Surgeons (ESES). Langenbeck's Arch Surg. 2014:399:155-63.

8. Viola D, Materazzi G, Valerio L, Molinaro E, Agate L, Faviana P, et al. Prophylactic central compartment lymph node dissection in papillary thyroid carcinoma: clinical implications derived from the first prospective randomized controlled single institution study. J Clin Endocrinol Metab. 2015;100:1316-24

9. Ito Y, Miyauchi A, Masuoka H, Fukushima M, Kihara M, Miya A. Excellent prognosis of central lymph node recurrence-free survival for cNOMO papillary thyroid carcinoma patients who underwent routine prophylactic central node dissection. World J Surg. 2018

10. Amin MB, Edge S, Greene F, Byrd DR, Brookland RK, Washington MK, et al AJCC Cancer Staging Manual. Eight edition. 2017; Springer International Publishing, New York, NY,: 872-927.

11. Hughes DT, White ML, Miller BS, Gauger PG, Burney RE. Doherty GM influence of prophylactic central lymph node dissection on postoperative thyroglobulin levels and radioiodine treatment in papillary thyroid cancer. Surgery. 2010;148:1100-6 discussion 006-7.

12. Bonnet $\mathrm{S}$, Hartl D, Leboulleux S, Baudin E, Lumbroso JD, Al Ghuzlan A, et al. Prophylactic lymph node dissection for papillary thyroid cancer less than 2 $\mathrm{cm}$ : implications for radioiodine treatment. J Clin Endocrinol Metab. 2009;94: $1162-7$

13. Zhao W, You L, Hou X, Chen S, Ren X, Chen G, et al. The effect of prophylactic central neck dissection on Locoregional recurrence in papillary thyroid Cancer after Total thyroidectomy: a systematic review and metaanalysis : PCND for the Locoregional recurrence of papillary thyroid Cancer. Ann Surg Oncol. 2017;24:2189-98.

14. Chen L, Wu YH, Lee CH, Chen HA, Loh EW, Tam KW. Prophylactic central neck dissection for papillary thyroid carcinoma with clinically uninvolved central neck lymph nodes: a systematic review and meta-analysis. World J Surg. 2018:42:2846-57.

15. Zetoune T, Keutgen $X$, Buitrago D, Aldailami $H$, Shao H, Mazumdar M, et al. Prophylactic central neck dissection and local recurrence in papillary thyroid cancer: a meta-analysis. Ann Surg Oncol. 2010;17:3287-93.

16. Wang TS, Cheung K, Farrokhyar F, Roman SA, Sosa JA. A meta-analysis of the effect of prophylactic central compartment neck dissection on locoregional recurrence rates in patients with papillary thyroid cancer. Ann Surg Oncol. 2013;20:3477-83. 
17. Kim MK, Mandel SH, Baloch Z, Livolsi VA, Langer JE, Didonato L, et al Morbidity following central compartment reoperation for recurrent or persistent thyroid cancer. Arch Otolaryngol Head Neck Surg. 2004;130: 1214-6.

18. Clayman GL, Agarwal G, Edeiken BS, Waguespack SG, Roberts DB, Sherman SI. Long-term outcome of comprehensive central compartment dissection in patients with recurrent/persistent papillary thyroid carcinoma. Thyroid. 2011:21:1309-16.

19. Al-Saif O, Farrar WB, Bloomston M, Porter K, Ringel MD, Kloos RT. Long-term efficacy of lymph node reoperation for persistent papillary thyroid cancer. J Clin Endocrinol Metab. 2010;95:2187-94.

20. Shen WT, Ogawa L, Ruan D, Suh I, Kebebew E, Duh QY, et al. Central neck lymph node dissection for papillary thyroid cancer: comparison of complication and recurrence rates in 295 initial dissections and reoperations. Arch Surg. 2010;145:272-5.

21. Schuff KG, Weber SM, Givi B, Samuels MH, Andersen PE, Cohen JI. Efficacy of nodal dissection for treatment of persistent/recurrent papillary thyroid cancer. Laryngoscope. 2008;118:768-75.

22. Stavrakis Al, Ituarte PH, Ko CY. Yeh MW surgeon volume as a predictor of outcomes in inpatient and outpatient endocrine surgery. Surgery. 2007;142: 887-99; discussion -99.

23. Sosa JA, Bowman HM, Tielsch JM, Powe NR, Gordon TA, Udelsman R. The importance of surgeon experience for clinical and economic outcomes from thyroidectomy. Ann Surg. 1998;228:320-30.

24. Kuo EJ, Thi WJ, Zheng F, Zanocco KA, Livhits MJ, Yeh MW. Individualizing surgery in papillary thyroid carcinoma based on a detailed sonographic assessment of Extrathyroidal extension. Thyroid. 2017;27:1544-9.

25. Roh JL, Kim JM, Park Cl. Central lymph node metastasis of unilateral papillary thyroid carcinoma: patterns and factors predictive of nodal metastasis, morbidity, and recurrence. Ann Surg Oncol. 2011;18:2245-50

26. Lee DW, Ji YB, Sung ES, Park JS, Lee YJ, Park DW, et al. Roles of ultrasonography and computed tomography in the surgical management of cervical lymph node metastases in papillary thyroid carcinoma. Eur J Surg Oncol. 2013;39:191-6.

27. Raffaelli M, De Crea C, Sessa L, Fadda G, Bellantone C, Lombardi CP. Ipsilateral central neck dissection plus frozen section examination versus prophylactic bilateral central neck dissection in cNO papillary thyroid carcinoma. Ann Surg Oncol. 2015;22:2302-8.

28. Yan X, Zeng R, Ma Z, Chen C, Chen E, Zhang X, et al. The utility of sentinel lymph node biopsy in papillary thyroid carcinoma with occult lymph nodes. PLoS One. 2015;10:e0129304.

29. Barczynski M, Konturek A, Stopa M, Nowak W. Prophylactic central neck dissection for papillary thyroid cancer. Br J Surg. 2013;100:410-8.

\section{Publisher's Note}

Springer Nature remains neutral with regard to jurisdictional claims in published maps and institutional affiliations.

Ready to submit your research? Choose BMC and benefit from:

- fast, convenient online submission

- thorough peer review by experienced researchers in your field

- rapid publication on acceptance

- support for research data, including large and complex data types

- gold Open Access which fosters wider collaboration and increased citations

- maximum visibility for your research: over $100 \mathrm{M}$ website views per year

At $\mathrm{BMC}$, research is always in progress.

Learn more biomedcentral.com/submissions 